\title{
Correction to: Rearrangements of Open Magnetic Flux and Formation of Polar Coronal Holes in Cycle 24
}

\author{
E.M. Golubeva ${ }^{1}$ (D) A.V. Mordvinov ${ }^{1}$ (D)
}

Published online: 6 December 2017

(c) Springer Science+Business Media B.V., part of Springer Nature 2017

\section{Correction to: Solar Phys \\ DOI 10.1007/s11207-017-1200-6}

Due to an error during processing the Electronic Supplementary Material (ESM) supplied by the author was not included with the article. Please find in this correction document the reference to the ESM.

The online version of the original article can be found under doi:10.1007/s11207-017-1200-6.

Electronic supplementary material The online version of this article

(doi:10.1007/s11207-017-1219-8) contains supplementary material, which is available to authorized users.

\footnotetext{
$凶$ A.V. Mordvinov avm@iszf.irk.ru

E.M. Golubeva golubeva@iszf.irk.ru

1 Institute of Solar-Terrestrial Physics of Siberian Branch of Russian Academy of Sciences, Lermontov st., 126a, Irkutsk 664033, Russia
} 